\title{
On the quantization accuracy of the acoustoelectric current
}

\author{
Xiang-Bai Chen, ${ }^{1,2, *}$ Xiang-Song Chen, ${ }^{1, \dagger}$ and Jie Gao ${ }^{1}$ \\ ${ }^{1}$ Department of Physics, Sichuan University, Chengdu 610064, China \\ ${ }^{2}$ Department of Physics, Ewha Womans University, Seoul 120-750, South Korea
}

(Dated: October 28, 2018)

\begin{abstract}
By deriving analytical formulae for the quantization accuracy of the acoustoelectric current, we reveal that: 1) the flatness of the current plateau for the typical present devices has reached the theoretical limit of about 100ppm over a 1/1000 change of the gate voltage; 2) increasing the transport channel length, and counterintuitively, increasing the acoustic wavelength as well, would improve the quantization accuracy, and very promisingly up to $0.01 \mathrm{ppm}$ as required for a quantum current standard.

PACS numbers: $73.50 . \mathrm{Rb}, 73.63 . \mathrm{Kv}$
\end{abstract}

Introduction: The observation of a quantized electric current driven by surface acoustic wave (SAW) in 1996 [1] visualizes a possible quantum current standard. The device comprises a split gate build on a GaAs-AlGaAs heterostructure containing a two-dimensional electron gas (2DEG). A negative voltage $V_{g}$ applied to the split gate depletes the 2DEG beneath the gate and leaves a onedimensional (1D) transport channel. When pinched off, the 1D channel becomes a saddle-like potential barrier. At large wave amplitude, the SAW-induced potential minima become moving quantum dots (QDs), which capture electrons within the dots and drag them through the potential barrier. The important finding of Ref. [1] is that such acoustoelectric current displays plateaux over certain range of $V_{g}$ and/or SAW power, and the plateaux fit well into the quantized expression $I=N e f_{\mathrm{SAW}}$, with $N$ an integer, $e$ the electron charge, and $f_{\text {SAW }}$ the SAW frequency in the $\mathrm{GHz}$ band.

Qualitatively, this quantization phenomenon is explained by the Coulomb-blockade effect: At a certain depth the QD can only hold a fixed number of electrons, because excess occupation is prohibited by significant Coulomb repulsion among electrons within the small QD. The detailed electron transport process and possible error mechanism in such devices have been studied theoretically but quite controversially, in both classical dynamics [2] and quantum mechanism [3, 4, 5, 6, 7]. Sometimes, a very accurate current quantization (better than $10^{-10}$ ) is predicted [6]. However, the flatness of the current plateau achieved in the original [1] and subsequent experiments [8, 9, 10, 11, 12, 13] never goes beyond $\sim 100 \mathrm{ppm} / 10^{-3} V_{g}$, far below the level of $\sim 10^{-8}$ required in metrology.

In this paper, we aim at a clear clue on what factors determine the quantization accuracy of the acoustoelectric current. We start with a novel analysis of the electron transport process and possible error mechanisms by

\footnotetext{
*Electronic address: xchen@ewha.ac.kr

${ }^{\dagger}$ Electronic address: cxs@scu.edu.cn
}

clarifying on the different mechanisms for electrons to leave a QD. Then by some approximation techniques, we derive analytical formulae for the quantization accuracy. These formulae reveal clearly the limitation of the typical present devices and the promising possibility of improving the accuracy for metrological use.

The electron transport process and error mechanisms: Since the quantization of acoustoelectric current is essentially the quantization of electron number in each $\mathrm{QD}$, we find it very illuminating to first study how the electrons can escape a QD. We clarify that there are three such escape mechanisms when the QD climbs up the potential barrier:

(i) directly flowing out when the QD reduces its depth;

(ii) back tunneling through the rear wall of the $\mathrm{QD}$;

(iii) non-adiabatic transition into excited states, followed by an easier tunneling.

Mechanism (i) occurs because the depth of the QD depends on the slope of the potential barrier, which is not uniform and typically has a maximum at the waist of the barrier. For mechanism (iii), the excited state can in principle be a free state so that the electron escapes the QD directly, but we focus on the dominant case of transiting to a higher-energy bound state in the QD, and the electron escapes via an enhanced tunneling probability.

With the above clarification, we divide the electron transport process through the potential barrier into three segments, characterized by the location of the QD:

(a) before the steepest $(\mathcal{S})$ point of the barrier;

(b) from the $\mathcal{S}$ point to the top of the barrier;

(c) after the top of the barrier.

In segment (a), all three escape mechanisms are in effect. The QD becomes shallowest at the $\mathcal{S}$ point, so in segment (b) electrons can no longer flow out directly and only mechanisms (ii) and (iii) remain. Segment (c) is irrelevant for us because all escape mechanisms effectively kick the electrons into the drain instead of the source. 
Let us denote $N_{X}$ as the QD occupation number at the entrance of the $1 \mathrm{D}$ channel, $N_{\mathcal{S}}$ as that at the $\mathcal{S}$ point, and $N_{T}$ as that at the top of the barrier. As we explained above, $N_{T}$ can be take as the final number of transported electrons per QD, so the acoustoelectric current is

$$
I=N_{T} e f_{\mathrm{SAW}}
$$

Variation in $N_{T}$ means an error in the current quantization. It should be noted that $N_{T}$ can only differ slightly from $N_{\mathcal{S}}$, by the "mild" escape mechanisms (ii) and (iii). In contrast, $N_{\mathcal{S}}$ may differ dramatically from $N_{X}$, because in segment (a) the QD reduces its depth greatly, so that electrons can escape in a "wild" manner of flowing out directly. We write

$$
N_{T}=\left(1-P_{t}-P_{v}\right) N_{\mathcal{S}}
$$

Here $P_{t}$ is the (small) probability for an electron to escape the QD in segment (b) by direct tunneling, and $P_{v}$ is the (small) probability for an electron to first transit to excited states by non-adiabatic correction, then escape the QD by an easier tunneling due to higher energy. From Eq. (2), we can define two additive error mechanisms in the quantization of $I=N_{T} e f_{\mathrm{SAW}}$. First, the "wild" reduction from $N_{X}$ to $N_{\mathcal{S}}$ could be inaccurate, which results in an indefinite $N_{\mathcal{S}}$. Second, even if $N_{\mathcal{S}} \equiv 1$, viz., exactly one electron remains in each QD at the $\mathcal{S}$ point, this single electron may still escape the QD in segment (b) by non-zero $P_{t}$ and $P_{v}$.

Investigation of the "wild" reduction from $N_{X}$ to $N_{\mathcal{S}}$ necessarily involves many-electron dynamics. It has been calculated numerically with classical dynamics in Ref. [2]. And quantum mechanically, Ref. [5] has performed a phenomenological study of this process at the very entrance to the $1 \mathrm{D}$ channel. In the following, we concentrate on the "mild" reduction from $N_{\mathcal{S}}$ to $N_{T}$, starting with $N_{\mathcal{S}}=1$, which is then just a one-particle problem. Experimentally, the first current plateau (corresponding to one electron transfer per SAW cycle) usually has the best quality. As we will see below, the escape probability $\left(P_{t}+P_{v}\right)$ alone explains the observed quantization accuracy very well. This means that the error in the reduction from $N_{X}$ to $N_{\mathcal{S}}$ is at most of the same order as $\left(P_{t}+P_{v}\right)$.

Back tunneling of electrons out of a moving QD has been studied numerically in Refs. [3, 4, 7]. Here, we develop some approximation methods which allow us to estimate this effect analytically. Let us first look at the tunneling probability given by the familiar WKB formula:

$$
P=\exp \left[-\frac{2}{\hbar} \int_{x_{1}}^{x_{2}} d x \sqrt{2 m^{*}(V(x)-E)}\right] .
$$

Here $m^{*}=0.067 m_{e}$ is the effective electron mass, $V(x)$ describes the QD rear wall to penetrate, $E$ is the electron energy, and $x_{1,2}$ are the two transition points at which
$V(x)=E$. It should be noted that $P$ in Eq. (3) is derived as a transmission coefficient, viz., the intensity ratio of the out-going and incident beams. In other words, $P$ is the penetration probability per collision. If an electron captured in a QD collides with the QD rear wall $\Gamma$ times per unit time, then the total probability for it to tunnel out of the $\mathrm{QD}$ in a time duration $\left[t_{1}, t_{2}\right]$ is

$$
P_{t}=\int_{t_{1}}^{t_{2}} P \Gamma d t
$$

The collision frequency $\Gamma$ is just the electron oscillation frequency in the QD. If we approximate the electron motion in the QD as a harmonic oscillator with angular frequency $\omega_{e}$, then $\Gamma=f_{e}=\omega_{e} / 2 \pi$, which is typically much higher than the SAW frequency $f_{\mathrm{SAW}}$. Evidently, Eq. (4) is equally valid if $P$ and $\Gamma$ vary with time.

Eq. (3) tells us that the back-tunneling probability $P$ is largely determined by the height and width of effective part of the QD rear wall lying above the electron energy. To examine these parameters, we make a reasonable approximation by considering the superposition of a sinusoidal potential wave $A \sin (2 \pi x / \lambda)$ and a straight potential slope $\kappa x$, with $\kappa$ corresponding to the local slope of the real potential barrier. The largest derivative of the sinusoidal wave, $2 \pi A / \lambda$, must exceeds $\kappa$, otherwise no QD can be formed. In a recent paper [14], the SAWinduced wave amplitude $A$ was estimated to be $25 \mathrm{meV}$. In contrast, to make $N_{\mathcal{S}}=1$ the depth $\Delta$ of the QD at the $\mathcal{S}$ point must be smaller than the charging energy of the QD, which is estimated to be $\sim 1 \mathrm{meV}[\underline{5}, 14]$. Since now $\Delta \ll A$, the slope $\kappa_{0}$ at the $\mathcal{S}$ point must be very close to $2 \pi A / \lambda$ so as to produce a superposed QD much shallower than $A$. We therefore define

$$
\frac{\kappa_{0}}{2 \pi A / \lambda} \equiv 1-\eta
$$

with $\eta$ a small parameter. Then, a slight algebra shows that the depth $\Delta$ of the QD is approximated by

$$
\Delta \simeq \frac{3}{\sqrt{2}} \sqrt{\eta^{3}} A,
$$

and the effective width of the QD rear wall is approximated by

$$
W \simeq \frac{3 \sqrt{2}}{2 \pi} \sqrt{\eta} \lambda .
$$

By symmetry, $W$ is also the effective diameter of the QD. We see that at the $\mathcal{S}$ point of the potential barrier, the size of the QD is much smaller than the SAW wavelength.

The parameter $\eta$ is not free. It can be fixed as follows: To permit only one electron in the QD, its depth $\Delta$ must not exceed the charging energy $E_{c} \sim e^{2} / r \epsilon_{\text {eff }}$, with $r=$ $W / 2$ the effective radius of the $\mathrm{QD}$ and $\epsilon_{\mathrm{eff}}$ the effective dielectric constant in the QD. From $\Delta \leq E_{c}$, we have

$$
\eta \leq \sqrt{\frac{4 \pi}{9 A \lambda} \frac{e^{2}}{\epsilon_{\mathrm{eff}}}} .
$$


The upper limit corresponds to the best quantization accuracy. To have a quantitative feeling of these parameters, take $A=25 \mathrm{meV}, \lambda=1 \mu \mathrm{m}$, and tentatively $\epsilon_{\text {eff }}=13$, we find

$$
\eta \leq 0.079, \quad \Delta \leq 1.2 \mathrm{meV}, \quad W \leq 0.19 \mu \mathrm{m} .
$$

They are all consistent with our preassumptions.

There is a hidden subtlety in Eq. (8) which calls for special attention: the choice of $\epsilon_{\text {eff }}$. Quite often, it is argued that since the 2DEG resides very close to the surface, (viz., with a distance $d \sim 0.1 \mu \mathrm{m}$ much smaller than the SAW wavelength) $\epsilon_{\text {eff }}$ is roughly half of the value for a body material. However, Eq. (9) indicates that the size of the QD is also much smaller than the SAW wavelength, and actually comparable to $d$, thus within the $\mathrm{QD} \epsilon_{\mathrm{eff}}$ can be close to the value $(\simeq 13)$ for a body material.

To apply Eq. (3), we still need the electron energy inside the QD. We approximate the electron motion as a harmonic oscillator with an angular frequency $\omega_{e}$ :

$$
m^{*} \omega_{e}^{2}=A\left(\frac{2 \pi}{\lambda}\right)^{2} \sqrt{1-\left(\frac{\kappa_{0}}{2 \pi A / \lambda}\right)^{2}} \simeq A\left(\frac{2 \pi}{\lambda}\right)^{2} \sqrt{2 \eta} .
$$

A quick order-of-magnitude estimation of the tunneling probability can be obtained by approximating the QD rear wall as a triangle with height $\Delta$ and width $W$ :

$$
P \sim \exp \left[-\frac{2}{\hbar} \cdot \frac{2}{3}\left(\frac{\Delta-E}{\Delta} W\right) \sqrt{2 m^{*}(\Delta-E)}\right] .
$$

Here $E \simeq\left(n+\frac{1}{2}\right) \hbar \omega_{e}$ is the electron energy relative to the bottom of the QD, and the ground state has $n=0$.

The final ingredient needed to compute $P_{t}$ is an explicit expression of the potential barrier. We take it to be

$$
V(x)=V_{0} / \cosh ^{2}(x / L) .
$$

The effective width of this potential barrier is $2 L$, the steepest point is at $x_{0} \simeq-0.66 \mathrm{~L}$, with a slope

$$
\kappa_{0} \equiv C_{1} V_{0} / L \simeq 0.77 V_{0} / L .
$$

The total probability for an electron to tunnel out in segment (b) is then

$$
P_{t}=\int_{x_{0}}^{0} P(x) \frac{\omega_{e}}{2 \pi} \frac{d x}{v_{s}},
$$

where $v_{s} \simeq 2800 \mathrm{~m} / \mathrm{s}$ is the SAW speed.

There are three free parameters in the above expressions: the SAW wavelength $\lambda$, the barrier width $2 L$, and the barrier height $V_{0}$. From Eqs. (5, [13), the tunneling probability at the $\mathcal{S}$ point can be expressed as

$$
P \sim \exp \left[-C_{t} \lambda^{\frac{1}{4}} L^{\frac{1}{8}} V_{0}^{-\frac{1}{8}}\left(1-C_{e} \lambda^{-\frac{1}{4}} L^{-\frac{1}{8}} V_{0}^{\frac{1}{8}}\right)^{\frac{3}{2}}\right] .
$$

This is our first master equation. Here $C_{t}$ and $C_{e}$ are straightforwardly computed coefficients independent of $\lambda, L$, and $V_{0}$. We postpone discussing the implications of Eq. (15), and turn to the other error mechanism:

Non-adiabatic excitation: This effect can be most conveniently studied by adopting a coordinate system comoving with the SAW. Because the potential barrier is not uniform, even in this co-moving coordinate system the electron feels a varying external field, which can lead to quantum transitions. Namely, an electron in state $a$ at time $t=0$ has a non-zero probability $P_{b a}(t)$ to be in another state $b$ at a later time $t$. To first order, $P_{b a}(t)$ is given by [15]

$$
P_{b a}(t) \simeq 4 \hbar^{-2} \omega_{b a}^{-4}\left|\left\langle\frac{\partial H}{\partial t}\right\rangle_{b a}\right|^{2} \sin ^{2}\left[\omega_{b a} t / 2\right] .
$$

Here $\omega_{b a}(t)=\left[E_{b}(t)-E_{a}(t)\right] / \hbar$, with $E_{a, b}(t)$ eigenvalues of the instantaneous Hamiltonian $H(t) .\left\langle\frac{\partial H}{\partial t}\right\rangle_{b a}$ is the matrix element of $\frac{\partial H}{\partial t}$ between the states $b$ and $a$. Because the electron oscillation frequency is much higher than the SAW frequency, the factor $\sin ^{2}\left[\omega_{b a} t / 2\right]$ in Eq. (16) oscillates very quickly. By approximating the electron motion in the QD as a harmonic oscillator, our problem resembles the textbook example of a charged harmonic oscillator in a time-dependent electric field [15], for which only the transition into the first excited state is relevant, and the transition probability $P_{10}$ can be expressed as

$$
P_{10} \simeq\left(\frac{v_{s}}{v_{e}}\right)^{2}\left(\frac{1}{m^{*} \omega_{e}^{2}} \frac{d^{2} V}{d x^{2}}\right)^{2} \sin ^{2}\left[\omega_{e} t / 2\right] .
$$

Here $v_{e} \equiv \sqrt{2 \hbar \omega_{e} / m^{*}}$ is an effective electron speed in the QD, with $\omega_{e}$ from Eq. (10). To see the dependence of $P_{10}$ on the experimental parameters, we write

$$
d^{2} V / d x^{2} \equiv C_{2} V_{0} / L^{2}
$$

with the coefficient $C_{2}$ independent of $V_{0}$ and $L$. Following the same steps as in deriving Eq. (15), $P_{10}$ can be expressed as

$$
P_{10} \simeq C_{v} \lambda^{\frac{15}{4}} L^{-\frac{17}{8}} V_{0}^{\frac{1}{8}} \sin ^{2}\left[\omega_{e} t / 2\right] .
$$

This is our second master equation. Here $C_{v}$ is another coefficient independent of $\lambda, L$, and $V_{0}$.

Quantization accuracy for the present devices: We now plug in explicit parameters to estimate the theoretical limit of the quantization accuracy of the acoustoelectric current. In a typical experiment [1, 8, 9, 10, 11, 12, 13], one has $\lambda=2 L=1 \mu \mathrm{m}$. There is just one parameter to tune: the gate voltage (which determines the barrier height $V_{0}$ ) or the SAW amplitude. But this parameter does not bring much freedom either. $V_{0}$ must be at least several tens of $\mathrm{meV}$ in order to be well above the electron Fermi energy. Furthermore, our two master equations 
(15) and (19) show a rather week dependence on $V_{0}$. For the experimentally estimated $A=25 \mathrm{meV}$ [14], Eqs. (5) and (13) give $V_{0} \simeq 94 \mathrm{meV}$, in consistent with our expectation. Then, Eq. (11) or (15) gives the tunneling probability of the ground-state electron at the $\mathcal{S}$ point: $P_{0} \sim 10^{-3}$, and Eq. (14) gives the total probability for this electron to tunnel out in segment $(\mathrm{b}): P_{t} \simeq 1.3 P_{0}$. This integrated tunneling probability is of the same order as the tunneling probability at the $\mathcal{S}$ point because $P$ drops very quickly as $x$ moves away from the $\mathcal{S}$ point.

The situation for non-adiabatic transition requires some caution. For an electron to eventually escape the QD by this mechanism, the transition must be followed by an easy tunneling. The tunneling probability is largest at the $\mathcal{S}$ point, but Eq. (17) says that the transition probability is related to the second derivative $d V^{2} / d x^{2}$, which is zero at the $\mathcal{S}$ point. At first thought this seems to imply that the non-adiabatic mechanism is not relevant. However, we note that our whole discussion for non-adiabatic transition can as well be applied to the center-of-mass motion of the interacting electrons in segment (a), where $d^{2} V / d x^{2}$ can be significant. This means that during this period the center-of-mass motion of electrons may transit to an excited state, by a probability of the same order as given by Eq. (17). The excited electrons will then escape the QD by the significant tunneling probability near the $\mathcal{S}$ point, leading to an error in current quantization. Therefore, to safely control this kind of quantization error, we should estimate $P_{v}$ as the product of the maximum $P_{10}$ in segment (a) with $P_{1}$ at the $\mathcal{S}$ point, where $P_{1}$ is the tunneling probability of the electron in the first excited state. A slight calculation with Eq. (17) gives $P_{10} \sim 2 \times 10^{-4}$, and Eq. (11) gives $P_{1} \sim 0.5$. Note that the WKB formulae is only valid at very small tunneling probability, therefore a $P_{1} \sim 0.5$ should be effectively taken as one (viz., $100 \%$ electrons escape). So we finally estimate $P_{v}$ in Eq. (2) to be $\sim 2 \times 10^{-4}$.

Putting $P_{t}$ and $P_{v}$ together, we see that the acoustoelectric current at the first plateau deviates from the quantized value $I_{1}=e f_{\mathrm{SAW}}$ by at least $1.5 \times 10^{-3}$. This absolute deviation is hard to detect due to the zero-point drift in measuring the electric current. What is easier to detect is the slope of the current plateau. By fixing $A=25 \mathrm{meV}$ and varying $V_{0}$, the slope is computed to be $d I / d V_{0} \sim 10^{-4} I_{1} / 10^{-3} V_{0}$. Since $V_{0}$ is proportional to the gate voltage $V_{g}$, this means that the acoustoelectric current varies by $100 \mathrm{ppm}$ as $V_{g}$ varies by $1 / 1000$. This agrees very well with the best experimental result [9].

Tuning the parameters for better accuracy: From our two master equations (15) and (19), we see that the quantization accuracy of acoustoelectric current is essentially set by the device parameters $\lambda$ and $L$. A longer transport channel, viz, a wider potential barrier, always reduces the error in current quantization. And quite counterintuitively, a longer SAW wavelength may also be advan- tageous, because as $\lambda$ increases the tunneling probability falls exponentially while the non-adiabatic transition probability increases in a power law.

A larger $\lambda$ also brings a hidden benefit that the QD size may indeed considerably exceed $d$, the distance of the 2DEG to the surface, thus the effective dielectric constant reduces and the Coulomb-blockade effect ehances. E.g., if we take $\lambda=L=5 \mu \mathrm{m}, V_{0}=90 \mathrm{meV}$, and pre-assume that $r=\frac{1}{2} W \ll d$ so that $\epsilon_{\text {eff }} \simeq 13 / 2$, we get $r \simeq 0.46 \mu \mathrm{m}$, in consistent with the preassumption. Proceeding with $\epsilon_{\mathrm{eff}} \simeq 13 / 2$, Eqs. (11) and (14) give $P_{0} \sim P_{t} \sim 10^{-14}$ and $P_{1} \sim 10^{-10}$, Eq. (17) gives $P_{10} \sim 10^{-3}$, and thus $P_{v} \sim 10^{-13}$. The slope of the current plateau is computed to be $d I / d V \sim 10^{-13} I_{1} / 10^{-3} V_{0}$. These accuracies are far enough for metrological use, which requires $0.01 \mathrm{ppm}$.

Summary: We performed a novel examination of the quantization accuracy of the acoustoelectric current. By clarifying the electron transport process and error mechanisms, we obtain a clear clue on the theoretical limit of the quantization accuracy, expressed by several analytical approximate formulae. We have shown unambiguously that the so far achieved quantization accuracy is already the theoretical limit of the present devices with $\lambda=2 L \sim 1 \mu \mathrm{m}$. To improve the accuracy to meet metrological requirement, it is necessary, and very promising, to have a larger $L$ and/or $\lambda$, e.g., of $5 \mu \mathrm{m}$.

This work is supported by the China NSF under Grant No. 60436010 and the State-Support Program of Science and Technology under Grant No. 2006BAF06B09.

[1] J. M. Shilton et al., J. Phys.: Condens. Matter 38, L531 (1996).

[2] A. M. Robinson and C. H. W. Barnes, Phys. Rev. B 63, 165418 (2001).

[3] G. R. Aǐzin, G. Gumbs, and M. Pepper, Phys. Rev. B 58, 10589 (1998).

[4] G. Gumbs, G. R. Aǐzin, and M. Pepper, Phys. Rev. B 60, R13954 (1999).

[5] K. Flensberg, Q. Niu, and M. Pustilnik, Phys. Rev. B 60, R16291 (1999).

[6] P.A. Maksym, Phys. Rev. B 61, 4727 (2000).

[7] H.Z. Guo, X.R. Chen, and J. Gao, Phys. Lett. A 359, 157 (2006).

[8] V. I. Talyanskii et al., Phys. Rev. B 56, 15180 (1997).

[9] J. Cunningham et al., Phys. Rev. B 60, 4850 (1999).

[10] N. E. Fletcher et al., Phys. Rev. B 68, 245310 (2003).

[11] K. Gloos, P. Utko, J. B. Hansen, and P. E. Lindelof, Phys. Rev. B 70, 235345 (2004).

[12] A.M. Robinson and V. I. Talyanskii, Phys. Rev. Lett. 95, 247202 (2005).

[13] M. Kataoka, C. H. W. Barnes, H. E. Beere, D. A. Ritchie, and M. Pepper, Phys. Rev. B 74, 085302 (2006).

[14] M.R. Astley et al., Phys. Rev. Lett. 99, 156802 (2007).

[15] See, e.g., B.H. Bransden and C.J. Joachain, Introduction to Quantum Mechanics (Longman, Essex 1989). 